CAHIERS DE

NARRATOLOGIE

\section{Cahiers de Narratologie}

Analyse et théorie narratives

29 | 2015

Street Art 1

\title{
Figures de l'anonymat. De quoi Banksy est-il le non ?
}

Une économie politique du visible

Marie-Joseph Bertini

\section{OpenEdition}

Journals

Electronic version

URL: http://journals.openedition.org/narratologie/7398

DOI: $10.4000 /$ narratologie. 7398

ISSN: 1765-307X

Publisher

LIRCES

Electronic reference

Marie-Joseph Bertini, «Figures de l'anonymat. De quoi Banksy est-il le non? », Cahiers de Narratologie [Online], 29 | 2015, Online since 08 January 2016, connection on 23 April 2019. URL : http://

journals.openedition.org/narratologie/7398; DOI : 10.4000/narratologie.7398

This text was automatically generated on 23 April 2019

Article L.111-1 du Code de la propriété intellectuelle. 


\section{Figures de l'anonymat. De quoi Banksy est-il le non?}

Une économie politique du visible

Marie-Joseph Bertini

1 J'ai choisi de m'interroger ici sur un street-artiste aimé, voire adulé, mais aussi détesté, notamment par un grand nombre de ses pairs qui entrevoient, dans sa démarche à la fois sinueuse et rectiligne, une certaine forme de cynisme. Pourtant Banksy, puisque c'est de lui qu'il s'agit, nous aide à percevoir et à comprendre des choses essentielles, bien au-delà de lui-même et de la représentation que nous en avons. Aussi dessinerai-je moins le portrait de Banksy en artiste engagé, que je n'essaierai de penser la manière dont il se dégage, s'extrait, se défile, s'exorbite de cette légende urbaine qu'il contribue à construire, mais qui fait aussi écran (au double sens du terme) à ce qu'il tend à nous donner à voir : une forme de célébration d'un art sans artiste, d'un message sans locuteur, d'une œuvre-manifeste qui congédie son auteur et nous transforme malgré nous en spectateurs émancipés. Un peu comme le lapin animé faisant disparaître son magicien, lequel constitue d'ailleurs une installation mise en place par Banksy en ce moment même, à DismaLand, son anti-parc d'attraction, créé avec l'aide d'une cinquantaine de complices du street art, dans une ville balnéaire décatie, près de Bristol ; un parc que l'on pourrait appeler de répulsion et dont la force narrative n'est pas sans rappeler les principes du théâtre épique brechtien.

2 Les notices Wikipédia du futur commenceront par : «Il est aujourd'hui établi avec certitude que l'artiste mondialement connu sous le pseudonyme Banksy, ... ». C'est cette certitude que Banksy nous refuse et c'est par elle qu'il désactive les frontières entre son art et sa portée sociale et politique, recréant, dans ses hétérotopies urbaines, l'espace de l'œuvre partagée et inattendue. De Tottenham à Gaza' ${ }^{1}$, en passant par Paris, Berlin, Los Angeles, la Palestine ou le Mali, Banksy fait le pari d'un art autonome qui apparaît là où personne ne l'attend et qui appartient par essence à tous. Le choix réaffirmé de son anonymat relève à mon sens d'une triple stratégie : esthétique, politique et éthique. Ce refus que Banksy oppose à tous ceux (et ils sont nombreux, tel ce tabloïd anglais qui mit sa tête à prix, ou bien cet internaute cherchant à vendre son identité aux enchères sur 
eBay $\left.{ }^{2}\right)$ qui cherchent à.... mais qui cherchent quoi exactement? Que cherche-t-on quand on veut nommer Banksy, le désigner par un autre nom que le pseudonyme ${ }^{3}$ qu'il s'est choisi, comme l'ensemble des street artistes avant lui ? Si l'anonymat renvoie à une personne, et plus particulièrement à un auteur, dont l'identité reste cachée, le pseudonyme renvoie lui au nom d'emprunt. Et cet anonymat est très relatif car, tout comme le Maître de Moulins, célèbre peintre français du XV'siècle dont l'identité a fait l'objet de nombreuses supputations, Banksy signe ses travaux sur les murs et édifices de nos villes (avec ces lettres, Banksy, ou bien avec l'emblème du rat, animal rejeté, invisible, anonyme et furtif, qu'il s'est choisi pour le représenter $\left.{ }^{4}\right)$; mais aussi avec sa patte, son style tellement reconnaissables qu'ils constituent autant de signatures avérées. De sorte que si Banksy demeure anonyme, ses œuvres sont, elles, parfaitement identifiées. Au Moyen-Age et à la Renaissance, l'anonymat d'un artiste renvoyait souvent à une notoriété telle, qu'elle rendait inutile le fait de signer l'œuvre, c'est-à-dire à la fois de la désigner comme œuvre et de revendiquer sa création.

Quelle importance, dès lors, que Banksy s'appelle en réalité « Robin Gunningham » ou bien « Robert Banks »? Qu'est-ce qu'un nom « vrai » et qu'est-ce qu'un artiste comme lui nous offre de comprendre en troquant son nom, contre un non ferme qui sonne comme une fin de non-recevoir, une négation dont les plis et les replis ouvrent à tous les possibles? Banksy argue de la nécessité de conserver cet anonymat pour se faufiler partout, empêcher qu'on le repère quand il se glisse dans les rues, dans les zoos, dans les musées, dans les décharges, dans ces espaces urbains que les street artistes contribuent à rendre vraiment publics. Liberté et sécurité sont mises en avant, et le street art, illégal à peu près partout dans le monde, a toujours eu partie liée avec la clandestinité et la subversion. D'autres street artistes connus conservent leur anonymat, tels le mosaïste Space Invader ou le belge Roa. Alors qu'est-ce qui rend l'anonymat de Banksy à la fois plus mystérieux et plus insupportable, sachant qu'il n'a pas inventé l'oxymore de l'inconnu célèbre et que sa posture n'est pas sans raviver le souvenir de l'irrévérence dadaïste?

4 Je ne peux m'empêcher à cet endroit de penser à deux cas plus éloignés de nous, mais similaires. Celui de Shakespeare, ou bien encore celui de Jack l'Eventreur. Que gagneraiton à savoir qu'ils s'appellaient James McCloud ou bien Timothy Simpson? Shakespeare et Jack l'Eventreur hantent nos mémoires et nos imaginaires comme des personnages à part entière dont le nom d'emprunt, le pseudonyme, fait signe vers la façon par laquelle notre mémoire collective agence les significations particulières qu'elle leur attribue (la poésie tragique et l'humanisme moderne d'un côté, l'effroi et l'horreur de l'autre). Dépouillés de leur «vrai » nom, ils ne sont plus des hommes, mais des fonctions par lesquelles ils tendent vers l'universel, aussi paradoxal que cela puisse paraitre dans le second cas. Où l'on peut voir ici que le nom, l'identité, ne permet pas l'identification. Comment reconnaître une pièce de Shakespeare ou bien un meurtre perpétré par Jack l'Eventreur ? Qu'est-ce qui fait qu'un Banksy est immédiatement reconnu comme tel aujourd'hui ? L'identification est étroitement reliée à la reconnaissance : celle de l'auteur (d'un crime, d'une pièce de théâtre ou d'une fresque murale), reconnaissance que l'on peut assimiler à l'ensemble des signes qui permettent d'authentifier une action, de la relier sans erreur à son véritable auteur.

5 De sorte que si Banksy s'évertue à rester anonyme ${ }^{5}$ (ce qui, dans une époque de transparence revendiquée comme la nôtre, s'apparente à une véritable gageure), ce n'est pas le cas de ses oeuvres qui, elles, sont signées, estampillées Banksy. Artiste en creux, 
œuvre en relief: ces oeuvres ont une identité, au sens où elles sont immédiatement reliables à lui. Ce sont des Banksy, comme on dirait des Picasso ou des Cézanne. Oui, mais non. Parce qu'on sait que Picasso et Cézanne s'appelait « vraiment » Picasso et Cézanne. Qu'est-ce que ce « vraiment » veut dire encore ? Qu'est-ce qui résiste là ? Banksy est-il le Daft Punk du street art (une stratégie marketing comme une autre, après tout), ou bien $y$ a-t-il autre chose, et si oui, de quoi s'agit-il ? Autrement dit qu'est-ce qui agit dans le non au nom de Banksy? Qu'est-ce qui se fait jour à l'endroit de cette négation et serait empêché de surgir à la lumière de son « vrai » nom?

6 Est « vrai » le nom qui éclaire un parcours, renvoie aux origines psychologiques, sociales, idéologiques, géographiques, familiales. Le « vrai » nom nous rive à une filiation qui pour être imaginaire, fait néanmoins pièce à la filiation artistique ou intellectuelle que l'on s'est choisie. Il enracine dans une histoire individuelle et commune, il réifie et objective celui qui le porte, interrompant, en l'individuant, le flux de toutes ses identités possibles. Le «vrai » nom institue l'origine en vérité, il fait de l'origine la vérité de celui qui le porte.

7 Oui, mais nous savons déjà tout ou presque de Banksy : que c'est un homme ${ }^{6}$, qu'il est né à Bristol, y a grandi dans les années soixante-dix, y a fait ses premiers graffs et a continué à le faire partout depuis. Ces détracteurs auraient-ils raison en affirmant que son anonymat est son meilleur agent auprès des publics spécialisés (galeristes, critiques, amateurs d'art, ...) et profanes? Que la médiatisation dont il bénéficie doit moins à son talent qu'à son sens aigü du buzz et des affaires? Après tout, Banksy dispose du relais de plusieurs sites internet très actifs, d'un agent, d'une attachée de presse, d'assistants, et il sait impliquer avec virtuosité les médias dans son dispositif esthétique (comme au mois de Février de cette année 2015 à Gaza). Ecoutons-le : «J'ai commencé à peindre dans la rue parce que c'était le seul endroit qui m'offrait un spectacle... Maintenant, je continue à peindre dans la rue pour me prouver que ce n'était pas un plan cynique ».

8 Le refus de Banksy de décliner son identité est aussi une façon, avancée par lui, de lutter contre la tentation ultime, celle de devenir un artiste reconnu et comme tel côté par le marché de l'art, élément contrôlable des dispositifs classiques de la reconnaissance : la galerie, l'exposition, le musée, les catalogues, qui sont les meilleurs moyens de soustraire un art à son topos originaire, en l'occurrence à la rue, pour lui octroyer un espace contingenté et codifié qui le vide de son sens initial et le réinvente en signaux de la modernité. Mais toute l'histoire de l'art est là, non seulement à travers les exemples proches de nous, ceux de Keith Haring ou de Basquiat, mais aussi ceux des frises du Parthénon Grec conservées au British Museum ${ }^{7}$, ou bien ceux des taureaux androcéphales ailés, gardiens des villes et palais assyriens, exposés au Louvre, ou encore de la sublime porte bleu d'Ishtar, autrefois à Babylone et aujourd'hui au Pergamon de Berlin, musée créé d'ailleurs pour recevoir les pièces monumentales transportées là à la faveur des guerres de conquête et des expéditions scientifiques (qui furent aussi l'autre nom des guerres de conquête). Toutes ces œuvres, dont la plupart des auteurs sont anonymes, ont été arrachées au lieu qui les avait produites et leur avait donné un sens originaire. A l'inverse du street art, contextuel par essence, qui réinvestit les lieux pour leur redonner un sens perdu dans les méandres des flux mondialisés, le propre du musée et de la conservation est d'inventer l'art hors sol, coupé de ses racines matérielles et spirituelles, et réinstallé au sein d'un réseau dense de significations à la fois nouvelles et universelles.

9 Depuis les années 2000, Banksy a lui-même disséminé ses travaux, de manière impromptue et clandestine ${ }^{8}$, au beau milieu des œuvres exposées dans les plus grands 
musées du monde (Tate Gallery, Moma, British Museum, Natural History Museum, Louvre). Si l'on reconnaît l'humour, la dérision, la provocation qui le caractérisent, on ne peut manquer d'y voir la conscience de ses propres paradoxes : comment être à la fois célèbre et inconnu, dedans et dehors, dans la rue et dans le musée, dans le système et hors de lui, au centre et à la marge ? En faisant le pari d'une œuvre vraiment ouverte, tout en détournement, en parodie, en substitutions, mais qui est aussi support de revendications d'ordre social et politique', l'anonymat de Banksy apparaît comme déterminant pour comprendre la place à la fois centrale et à part qu'il occupe sur la scène mondiale du street art. Par cet anonymat, l'art de Banksy ouvre sur une absence, un vide, une négation qui déroule « l'espace herméneutique ${ }^{10}$ » de ses créations. Ce lieu à partir duquel la geste de Banksy prend forme est un non-lieu, transitoire, éphémère, furtif et clandestin, et pour tout dire sauvage. Sans doute aussi parce qu'appuyé sur un fond de roussauïsme et de libertarisme, mâtiné de Justicier Masquée ${ }^{11}$, l'artivisme de Banksy cherche moins à réenchanter le monde qu'à le réensauvager ${ }^{12}$, à rendre animaux et humains à leur sauvagerie première, antidote selon lui à l'impérialisme panoptique de la publicité, du consumérisme, du pouvoir des forts sur les faibles et de la surveillance généralisée.

10 Banksy renouvelle les figures de l'anonymat dans l'art. Il ne s'agit plus seulement d'un anonymat convenu, protecteur ou confortable: le mensonge avoué du pseudo, qui remplace le nom par un signe, désigne ici l'illusion du vrai nom, de l'identité individuelle, stable et unique qui fait écran à l'œuvre et lui soustrait sa dimension mystérieuse, la part non visible de ce qu'elle donne à voir. Ce retrait et cette opacité sont garants de son efficience, de la manière dont elle creuse le présent pour y faire événement, pour inquiéter, questionner et échapper à ce qui la referme sur elle-même et la fige en œuvre. On peut penser ici au compositeur américain, John Cage, déclôturant et désacralisant ses œuvres en laissant des sons impromptus s'y introduire, à travers la porte laissée ouverte sur la rue pendant ses concerts, mettant ainsi en œuvre une dualité dehors/dedans, silence/sonorité où l'intention s'abolit dans le hasard et où l'art se transforme en une simple expérience de vie.

11 Pour expliquer le choix de son exposition/performance dans la ville de New-York durant tout le mois d'Octobre 2013, Banksy dit : « Je l'ai choisie pour son intense trafic de piétons et pour le nombre de lieux où se cacher ». En reliant ainsi le visible au caché, l'artiste installe le dissimulé au cœur même du perçu. Il faut rappeler en effet que Banksy ne masque pas seulement son identité, mais aussi les traits de son visage et le son de sa voix ${ }^{13}$ : il se gomme, se biffe, s'efface pour pouvoir travailler librement et partout. Et cela le rend paradoxalement omniprésent dans les esprits. Car si selon le philosophe anglais Berkeley : «être, c'est être perçu », être Banksy, c'est être imperceptible comme tel, de sorte qu'être un Banksy soit synonyme de la meilleure perception possible. Ce basculement incessant, tel un ruban de Moebius, du souterrain vers la surface, de l'occulté vers l'ostensible, de l'invu vers le vu, est stimulant. Connu parce qu'inconnu, célèbre parce qu'anonyme, Banksy se dévoile en même temps qu'il s'escamote et se fait disparaître : il est le lapin, le chapeau et le magicien à la fois. Il y a du Houdini chez Banksy.

12 Comme Thomas l'Obscur ${ }^{14}$, personnage central du roman de Maurice Blanchot que l'on ne connaît que par ce seul prénom et qui toujours se dérobe et s'absente, l'objet Banksy met en échec nos mouvements pour le saisir et la distance ainsi ouverte ne se laisse pas réduire, car elle est ce qui lui donne sa profondeur sensible et non sensible. Cacher pour 
ne rien dissimuler, occulter pour donner à voir, ces mouvements en apparence contradictoires dessinent une économie politique du visible par laquelle Banksy se saisit lui-même comme part invisible de son oeuvre, point aveugle présupposé par elle et échappant à tout définition. On songe à Albert Camus, visitant en 1958 une exposition d'Yves Klein, et y trouvant le titre d'un de ses articles intitulé « Avec le vide, les pleinspouvoirs ».

ce des œuvres de Banksy, homme sans qualités ${ }^{15}$, viendrait donc de sa manière de décliner le pouvoir de l'auteur, de l'aucteur faudrait-il écrire, de s'en déprendre, de troquer son autorité et ses privilèges contre l'assurance que son travail n'en sera que plus attendu, regardé, redouté. C'est une manière de se défaire d'une fonction dont Barthes a montré autrefois qu'elle était consubstantielle à celle du discours critique ${ }^{16}$, ajoutant qu' « il n'y a donc rien d'étonnant à ce que, historiquement, le règne de l'Auteur ait été aussi celui du Critique ${ }^{17}$ ». Cette «théologie » du sens, instaurée par la notion d'auteur, est la condition de l'institutionnalisation de l'œuvre, autrement dit de sa récupération dans la sémiosis générale, dans le système d'interprétation que le critique ou l'expert instaurent en assignant à l'œuvre un sens, en même temps qu'une origine. Il est intéressant de rapprocher ces éléments du film de Banksy, Faites le mur ${ }^{18}$ ! dans lequel il a choisi un angle d'approche totalement décalé où le sujet du film n'est pas Banksy, mais un personnage improbable, Thierry Guetta, l'anti-Banksy, devenu MBW, créature banksyenne s'il en fût. Banksy n'apparaît dans ce document que comme témoin, de manière latérale, adventice et fortuite. Il est invité dans son propre film et ne livre pas son point de vue, mais celui d'un autre sur Banksy, sur les street artistes et sur lui-même ${ }^{19}$.

14 Mais allons plus loin. Michel Foucault nous dit que le nom de l'auteur "manifeste l'événement d'un certain ensemble de discours, et il se réfère au statut de ce discours à l'intérieur d'une société et à l'intérieur d'une culture.... [il] n'est pas situé dans l'état civil des hommes, il n'est pas non plus situé dans la fiction de l'œuvre, il est situé dans la rupture qui instaure un certain groupe de discours et son mode d'être singulier... La fonction auteur est donc caractéristique du mode d'existence, de circulation et de fonctionnement de certains discours à l'intérieur d'une société ${ }^{20} »$. Pour Foucault, le nom de l'auteur, "cette catégorie fondamentale de la critique, "l'homme-et-l'œuvre ${ }^{21}$ " " est prétexte à des formations discursives en prise avec des formations institutionnelles. L'effacement de l'auteur en tant qu'auteur, quel que soit le nom qu'on lui donne, semble donc impossible dans la mesure où la fonction auteur est aussi celle qui rend possible le jugement esthétique, au sens kantien du terme. Désigner les productions de Banksy comme relevant de l'art, même s'il s'agit du street art, revient à réactiver cette fonction, opération par laquelle on «bloque ainsi le constat de disparition de l'auteur et retient en quelque sorte la pensée au bord de cet effacement ${ }^{22} »$.

15 Ce à quoi John Searle, qui s'interroge dans Les Actes de langage ${ }^{23}$ sur le sens et la valeur du nom propre, ajoute que ce dernier présuppose toujours l'interrogation sur l'identité de celui qui le porte, autrement dit que le principe d'identification gouverne son usage. Mais, écrit Foucault, «La vérité est tout autre : l'auteur [...] est un certain principe fonctionnel par lequel, dans notre culture, on délimite, on exclut, on sélectionne. Bref, le principe par lequel on entrave la libre circulation, la libre manipulation, la libre composition, décomposition, recomposition [...] L'auteur est donc la figure idéologique par laquelle on conjure la prolifération du sens ${ }^{24} »$. Pour être vraiment ouverte, une œuvre devrait pouvoir se délester de ces deux principaux opérateurs que sont la notion d'auteur et celle d'œuvre même, par quoi est affirmé son caractère singulier et rémanant. 
S'y ajoute la notion d'artiste qui est le produit de la combinaison des deux autres. L'idée d'un art sans artiste résonne moins ici comme un paradoxe, que comme un cadre programmatique au sein duquel l'anonymat actualise l'art de l'ère post-artistique ${ }^{25}$ et post-sociale ${ }^{26}$, et installe en son centre le triple principe de l'émancipation ${ }^{27}$, de l'équivoque et du partage, principe qui est peut-être l'une des meilleures définitions que l'on puisse donner de l'esthétique et de sa dimension expérimentale et exploratoire.

Cependant, le nom propre Banksy ne prend-il pas étrangement toute la place laissée libre, ne sature-t-il pas cet espace dont l'auteur est évacué ? Si le pseudonyme emplit l'espace déserté par l'auteur, que reste-t-il de sa volonté de conserver un anonymat dont on voit bien les contorsions auxquelles il doit se livrer pour le maintenir comme tel ? Banksy, cet habile mélange de Ken Loach, de Monty Python, de Culture Underground, de Bakounine et de Blek le Rat, est-il récupéré par Banksy? Poser les choses ainsi, c'est se donner les moyens d'analyser autrement la question du hors-champ, si importante et déterminante, notamment dans le domaine du street art que l'on peut désigner tout entier comme utopie du hors-champ. Il semble en effet que ce que Banksy nous aide à comprendre, c'est que la position hors-champ est intenable, autrement dit que le hors-champ n'existe plus, qu'il n'a peut-être jamais existé. L'origine du mot "champ " revêt un intérêt tout particulier. Il appartient à la culture médiévale et fait partie du vocabulaire héraldique : le champ figure dans le blason "un secteur délimité ». Au XVIIIe siècle, c'est avec l'optique que ce mot poursuivra son lent cheminement, désignant une portion de l'image enregistrée par l'œil. On le retrouve un peu plus tard dans le vocabulaire de la photographie et du cinéma, où il désigne cette fois la portion de l'image enregistrée par l'œil mécanique de la caméra: le champ, c'est l'échelle des plans qui décomposent l'image.

17 Le travail d'un street artiste en général, et de Banksy en particulier, relève d'une mise en abyme, de l'emboîtement des différents plans de lecture et d'interprétation, parmi lesquels la plupart du temps ${ }^{28}$ ceux-ci au moins : les graffs ${ }^{29}$ inscrits sur un support urbain en trois dimensions, la réception de ces graffs par les passants transformés en spectateurs, l'interaction volontaire ou involontaire de ces derniers avec eux ${ }^{30}$, leurs captations photographiques incluant ou non d'autres passants/spectateurs, leur réverbération dans les différents médias, l'analyse et la projection de ces graffs dans le cadre de pratiques discursives. Ces plans, enchâssés les uns dans les autres, forment ce que Merleau-Ponty appelait la profondeur du visible : « Mais le propre du visible [...] est d'être superficie d'une profondeur inépuisable : c'est ce qu'il fait qu'il peut être ouvert à d'autres visions que la nôtre ${ }^{31}$ ». C'est en ce sens que le champ peut être désigné comme surgissement de la profondeur du visible.

Se pose la question dès lors de savoir à quelle distance regarder une production de street art. Autrement dit, quel est le point de vue à partir duquel celle-ci prend forme et sens, alors même que le spectateur/passant devient aussitôt le contre-champ de l'image, qu'il y est embarqué et enrôlé, et cesse d'être ce point à partir duquel la vision s'organise ? Le hors-champ désigne alors ce lieu devenu impossible, intenable, parce qu'absorbé par la représentation ${ }^{32}$ et par ses multiples réverbérations qui forment l'image-système ou bien devrais-je dire l'image-monde, au sein de laquelle le paradigme de la représentation cède la place au paradigme de l'immersion ${ }^{33}$. Comment voir une image ${ }^{34}$ dans laquelle on se déplace et déambule sans cesse, la saisir comme telle, pour ne pas confondre le donné de la représentation avec le donné sensible lui-même, pour ne pas échanger le monde contre son simulacre? Peut-être en comprenant que le hors-champ de l'image n'est pas le réel, 
mais ce que l'art fait au réel, la manière dont il l'attire, l'aspire et l'absorbe pour le réinventer.

Banksy dit et répète que son anonymat l'aide à empêcher toute récupération par le marché de l'art et par ses corollaires. Or l'ensemble de la démarche de Banksy relève de la mise en scène de ce refus, de la scénographie de cette négation. Le non au nom de Banksy est à lui seul un théâtre démultiplié par le miroitement des médias et des commentaires. Avec Banksy, tout est désormais dans le champ. Même dehors, dans la rue, nous sommes dedans, pris dans la toile, dans le filet de ses compositions. Il devient metteur en scène total, comme durant ce fameux mois à New York ${ }^{35}$ où il transforme l'environnement de son travail en éléments actifs/passifs de son exposition: les spectateurs photographiant ses œuvres, les vigiles les protégeant, les policiers le traquant à travers le lacis des rues de la ville, les racketteurs exigeant d'être payés pour laisser le public photographier ou filmer, les internautes qui ont fait circuler les images sur le Web, les tenants scandalisés du respect de la propriété privée, les afficionados qui l'ont défendu et soutenu, les journalistes qui se sont faits l'écho de chacune de ses créations, ses détracteurs et ses ennemis qui les ont vandalisées, sans oublier ceux qui les découpent à la scie circulaire pour les emporter et les vendre aux enchères. Dès lors, pourrait-on dire que Banksy devient le seul point à partir duquel la vision et l'interprétation de l'ensemble de ces plans s'organise, ou bien forme-t-il lui-même, avec tous ses personnages, le théâtre de la cruauté banksyen, expression par laquelle Artaud désignait le prix à payer pour donner vie aux représentations, pour les incarner dans un art dilaté au point de déborder et d'englober le réel?

L'anonymat de Banksy joue ici un rôle essentiel: il lui permet de devenir, avec toute l'ironie et la distance qui sont les siennes, l'un de ses propres personnages, d'échapper à la tentation de tenir seul la position-sujet, qui est aussi celle du sujet de la perspective. Banksy nous oblige ainsi à nous demander : où se placer pour voir tout ce qu'il y a à voir ? Comment bien voir et quoi regarder? Comment voir ce que nous regardons? Les lois de la perspective optique se transforment alors en lois de la perspective sociale, célébration d'un art sans artiste, lointain écho d'une architecture sans architecte prônée par Habermas ${ }^{36}$. Le standpoint de Banksy apparait paradoxalement comme création d'un antipoint de vue, ou point de vue non-subjectif, sorte de mise à l'écart de la perspective et de la morale de la perspective : le point de vue du sujet n'est plus intéressant. C'est en quoi Banksy n'est pas un moderne, et incarne un sujet collectif contestataire, ou pour le dire autrement une forme singulière de subjectivation de la contestation et de la subversion. Le point de vue chez Banksy, c'est celui du donné à voir, composé par la fresque et son environnement ; il part du représenté et non du spectateur ou de l'auteur. Avec lui, les murs nous observent, ses graffs s'interrogent sur nous ${ }^{37}$ et sur eux-mêmes, tandis que nous les ignorons, comme ces passants qui cheminent près d'eux sans les voir.

Les choses, les animaux, les situations héritent chez lui d'un point de vue, prennent la parole et dialoguent entre eux, tout comme ce chien au pochoir, en train d'uriner sur une borne d'incendie qui lui dit, mezzo voce,: «tu me complètes ${ }^{38}$ ». Comme chez Francis Ponge, « le parti pris des choses $^{39}$ » est un élément central de la poétique banksyenne et avec lui, celui de la multiplication des sujets de l'expérience ${ }^{40}$. Pour Rancière, dans une communauté des égaux le régime esthétique se manifeste par une forme d'anonymat de l'art, c'est-à-dire par une expérience singulière du monde qui passe par un arrangement des objets, un point de vue qui vient de l'agencement des objets ${ }^{41}$. "Insaisissable dans l'immanence ${ }^{42}$, Banksy s'efface devant les choses et leur représentation, comme devant 
les causes qu'il défend, et sa présence-absence renvoie à ce qu'écrivait Pierre Klossowski dans les Lois de l'hospitalitét ${ }^{43}$ : "Fasciné par le nom de Roberte en tant que signe, alors que j'étais dans le jardin sans plus rien voir de l'ensoleillée verdure autour de moi, n'ayant d'autre vision que la pénombre insituable où se jouait la lueur de sa main dégantée - je me décide à décrire ce qui doit se passer dans cette pénombre, ici illusoire. Je réfère au nom de Roberte ce que je vois et que je ne verrais point à défaut de ce nom ». Que voyonsnous quand nous référons ce que nous voyons au pseudonyme Banksy, c'est-à dire au refus qui est le sien de se nommer? A quel événement ce signe, Banksy, renvoie-t-il ? Comment, de même que Klossowski, qui était aussi peintre et dessinateur, tente de le faire à travers Roberte, posséder ce qui se dérobe à toute possession, saisir l'insaisissable dans l'immanence et appréhender ce retrait comme présence? « Puisque l'absence est le lieu premier du discours ", disait Foucault ${ }^{44}$.

C'est en ce sens que Banksy est irrécupérable, qu'il échappe au foisonnement infini des pratiques discursives. C'est peut-être au fond ce que Debord voulait dire en parlant de société du spectacle. La société du spectacle, c'est sans doute celle qui abolit le horschamp, rendant la récupération impossible en mettant tout au même niveau : l'oeuvre, le mur, l'artiste, le spectateur, le capitalisme, le marché, la guerre, la paix ; ce qui supprime la possibilité même de l'extériorité, ou bien nécessite la fabrication incessante, chaplinesque, d'extériorités aussitôt assimilées par elle. Débarrassés du hors-champ, la subversion s'empare de la place laissée libre par une transgression désormais hors d'atteinte. Notons que les tentatives de récupération ne sont pas seulement d'ordre politique ou économique. Elles sont aussi académiques et savantes. Mais puisque nous venons de voir que ces tentatives de récupération sont des apories, je deviens moi-même au fond un personnage de l'oeuvre de Banksy en l'analysant, tout comme vous, qui me lisez aujourd'hui. Alors, quel effet ça fait d'être un Banksy?

Qu'est-ce qu'une représentation au juste, nous demande Banksy? N'est-ce pas ce qui est dotée de toutes les interprétations possibles, qui conserve son opacité et son mystère, son ambiguïté dirait Umberto Eco, parce je ne peux construire à partir d'elle un point de vue autre que celui de James Joyce dans Ulysse : labile, ondoyant, instable et aussitôt caduc? Ce faisant, Banksy montre qu'une représentation est toujours moins qu'une représentation, qu'elle est faite de ce qu'elle évide et retranche, mais aussi de ce qui s'en extrait volontairement pour la rendre autonome.

Comment se dégager de la triade qui menace alors: mystère/mystification/ mythification? En comprenant que le mystère de Banksy donne à voir tout ce qu'il cache, cours et jardins, scènes et coulisses. Klossowski encore : «La mystification contrefait le mystère et le présuppose $\mathrm{e}^{45} »$. Les formes subreptices et les codes du langage visuel, incisif et subversif, développés par Banksy, tout en détournements, en recyclages et en ironie provocatrice, dessinent un triple schéma conducteur: elles prônent une économie du don, fondés sur l'ouverture, la traduction et la transversalité, une esthétique de coup en instaurant une logique purement événementielle, une éthique de la ténacité par laquelle s'organisent des résistances et des solidarités, dont l'enjeu est profondément politique.

Cette perspective offre une grille de lecture pertinente de la post-modernité : elle traduit une éthique du changement, une morale du décentrement et du désaisissement, articulant l'anonymat de Banksy à un espace-mouvement dont les trajectoires multiples traduisent l'errance productive du vagabondage, ce cheminement vague qui construit sa question à partir des réponses jaillies du hasard et de la circonstance. Il est difficile de ne pas penser ici à l'opposition formulée par Michel de Certeau entre tactique et stratégie. 
"J'appelle "stratégie" le calcul des rapports de forces qui devient possible à partir du moment où un sujet de vouloir et de pouvoir est isolable d'un "environnement". Elle postule un lieu susceptible d'être circonscrit comme un propre... J'appelle au contraire "tactique" un calcul qui ne peut pas compter sur un propre... La tactique n'a pour lieu que celui de l'autre. Elle s'y insinue, fragmentairement, sans le saisir en son entier, sans pouvoir le tenir à distance. Elle ne dispose pas de base où capitaliser ses avantages, préparer ses expansions et assurer une indépendance par rapport aux circonstances ${ }^{46} »$.

Tacticien, Banksy, parce que passant outre la frontière du sujet et de l'objet ${ }^{47}$, de l'altérité et du propre, tentant de rendre son travail non capitalisable et non profitable, s'extirpant de l'auto-référentialité vaine d'un tout petit monde où les graffeurs parlent aux graffeurs, donnant la parole à ceux qui n'en sont pas dotés, utilisant le repérage, le jeu (de pistes), la dérision et l'inachevé comme antidotes à toutes les formes de captation. Que Banksy soit «bankable » et qu'il refuse de faire fructifier sa bankabilité en agace plus d'un...

Son anonymat jubilant, qui lui permet de se tenir loin de l'icône du street art et de l'artiste mainstream à quoi certains voudraient le réduire, est l'un des piliers de sa tactique : rusé, manipulateur, véloce, de mauvaise foi, exploratoire, silencieux, dispersé, parcellaire, invu, saisissant toutes les occasions face aux stratèges de l'art, quel que soit le plan sur lequel leurs stratégies produisent leurs effets. Cette esthétique - pourtant puérile et potache par moments - du canular, du pied-de-nez et du pied dans la porte, de l'attraction irrésistible de l'interdit, mais aussi de l'apparition/disparition, de l'ubiquité ${ }^{48}$ et de l'immanence comme horizon d'attente de l'artiste urbain, passeur et passant anonyme, fait écho à ces mots de La Fable mystique du même de Certeau : « Est mystique celui ou celle qui ne peut s'arrêter de marcher et qui, avec la certitude de ce qui lui manque, sait de chaque lieu et de chaque objet que ce n'est pas ça, qu'on ne peut résider ici ni se contenter de cela. Le désir crée un excès. Il excède, passe et perd les lieux. Il fait aller plus loin, ailleurs. Il n'habite nulle part ${ }^{49}$... ».

$\mathrm{Au}$ commerce des œuvres, Banksy tente de substituer le commerce des regards dont Mondzain nous dit le pouvoir de faire advenir un monde commun par la passion partagée des images ${ }^{50}$. Processus de requalification de l'œuvre d'art, dont elle révèle le mouvement interne et les glissements ininterrompus, la tactique de l'anonymat de l'artiste devient politique au sens où elle perpétue un art public que les médias, les critiques, les collectionneurs privés, les banquiers, les galeristes, les musées, sans oublier l'auteur de ce texte, échouent à s'approprier et à réduire à la fonction d'œuvre. Faire le mur, nous rappelle Banksy, c'est toujours à la fois s'en emparer et s'en échapper...

\section{BIBLIOGRAPHY}

BANKSY, (2010) Guerre et Spray, Paris : Gallimard, coll. Alternatives. Titre original : Wall and Piece.

BANKSY, (2011) Faites le mur ! Film, Paris : éditions France Télévision.

BARTHES, R. (1968) «La mort de l'auteur ». In: Revue Manteia, 5, 12-17. In: Oeuvres complètes, tome III (1968-1971), Livres, textes, entretiens, Paris, Le Seuil, 2002. 
BLANCHOT, M. (2005) Thomas l'Obscur, Paris : Gallimard.

CERTEAU de, $M$.

- (1990) Les arts de faire, tome 1, L'invention du quotidien, Paris : Gallimard.

- (1982) La fable mystique, Paris : Gallimard.

CHATEAU, D. (2011) « Les valeurs de l'art à l'ère du post-art ». In: Art, Emotion and Value, 5th Mediterranean Congress of Aesthetics, Carthagène.

FOUCAULT, M. (1969) «Qu'est-ce qu'un auteur?». In: Bulletin de la Société française de philosophie, 63ème année, 3, 73-104. In: Dits et Ecrits, I, Paris : Gallimard.

GADAMER, H. (1996) Vérité et Méthode, Paris : Le Seuil.

HABERMAS, J. (1999) Ecrits politiques, Paris : Flammarion.

KLOSSOWSKI, P. :

- (1995) Postface aux Lois de l'hospitalité, Paris : Gallimard.

- (1954) Roberte, ce soir, Paris : Gallimard.

MERLEAU-PONTY, M. (1964) Le visible et l'invisible, Paris : Gallimard.

MUSIL, R. (2004) L'homme sans qualités, tomes 1 et 2, Paris : Le Seuil.

RANCIERE, J. :

- (2008) Le spectateur émancipé, Paris : La Fabrique.

- (2003) Le destin de l'image, Paris : La Fabrique.

- (2000) Le partage du sensible. Esthétique et politique, Paris : La Fabrique.

SEARLE, J. (1972) Les actes de langage, Paris : Herman.

TOURAINE, A. (2013) La fin des sociétés, Paris : Le Seuil.

\section{NOTES}

1. En Février 2015. Ces créations font suite au Projet Santa's Ghetto, mené en Palestine en Juillet 2005.

2. Où l'on voit que l'anonymat de Banksy a non seulement une valeur, mais aussi un prix: l'enchère la plus haute a atteint près d'un million de dollars.

3. Le pseudonyme, appelé blaze, est la règle dans les milieux du street art, pour des raisons évidentes (c'est une pratique illégale) et moins évidentes (il est le véhicule de la réputation du street artiste, de la nouvelle identité qu'il s'est choisie).

4. Expression de l'une de ses nombreuses affinités, volontaires ou involontaires, avec un autre street artiste d'importance, Blek le Rat.

5. Cf. Banksy, Guerre et Spray, Paris, Gallimard, coll. Alternatives, 2010, p.29. Titre original de l'ouvrage: Wall and Piece.

6. Même si certains journalistes en mal de sensations ont prétendu le contraire.

7. Notons qu'une des installations clandestines de Banksy au British Museum a été maintenue après coup en bonne place par ce dernier.

8. Sur le principe du Hang and Run.

9. Accompagné de messages parfois simplistes et de raccourcis qui offrent une vision du monde plutôt binaire. 
10. cf. Hans Gadamer, Vérité et Méthode, Paris, Le Seuil, 1996.

11. L'une de ses fresques urbaines représente Zorro sur son cheval cabré, tenant un rouleau de peinture à la main.

12. cf. Le pochoir sur lequel, de sa démarche chaloupée, un tigre s'enfuit d'un code-barre transformé en camion-prison.

13. Modifiée par des techniques électroniques. Il est frappant en revanche qu'il donne à voir librement ses mains, comme s'il fallait faire un lien entre le visage effacé de l'artiste d'un côté et les mains offertes de l'artisan de l'autre, entre la mort de l'auctor et la célébration du faber.

14. Paris, Gallimard, 2005.

15. Cf. Robert Musil, L'homme sans qualités, tomes 1 et 2, Paris, Le Seuil, 2004. Ulrich, le personnage principal, représente pour Musil le paradigme de l'indétermination, de l'indéfinition et de l'ouverture.

16. Cf. «La mort de l'auteur », revue Manteia, 5, 1968, pp.12-17.

17. Roland Barthes, "La mort de l'auteur ", in Oeuvres complètes, tome III (1968-1971), Livres, textes, entretiens, Paris, Le Seuil, 2002, p. 44.

18. Titre original : Exit Through the Gift Shop.

19. De la même façon, son documentaire Antics Roadshow, diffusé en 2011 sur la chaîne Britannique Channel 4, était centré sur les pranksters, les provocateurs, comme le vidéaste Rémy Gaillard, les entarteurs ou les Yes Men.

20. Michel Foucault, "Qu'est-ce qu'un auteur?», Bulletin de la Société française de philosophie, 63ème année, $\mathrm{n}^{\circ}$ 3, Juillet-Septembre 1969, pp. 73-104, in Dits

21. Ibid., p.820

22. Ibid., p.823.

23. Paris, Herman, 1972.

24. Michel Foucault, op.cit., p.839.

25. cf. Dominique Chateau, "Les valeurs de l'art à l'ère du post-art ", Art, Emotion and Value, 5th Mediterranean Congress of Aesthetics, Carthagène, 2011.

26. cf. Alain Touraine, La fin des sociétés, Paris, Le Seuil, 2013.

27. cf. Jacques Rancière, Le spectateur émancipé, Paris, La Fabrique, 2008 et Le partage du sensible. Esthétique et politique, Paris, La Fabrique, 2000.

28. Le cas des projections vidéos ou des installations est similaire.

29. Les œuvres de Banksy, et des street artistes en général, étant multiformes, le terme générique graff désigne ici l'ensemble des productions esthétiques clandestines réalisées dans les espace publics, combinant ou non images et textes, et quel que soit le médium utilisé (pochoirs, affiches, collages, pastels, mosaïques, toiles peintes, installations,...). Si le graffiti au pochoir est aujourd'hui l'une des techniques les plus utilisées par Banksy, c'est aussi parce qu'elle lui permet de peindre avec précision et très rapidement des motifs complexes.

30. cf. la photographie du pochoir représentant un chien dessiné à la Keith Haring et aboyant sur une passante réelle. Près du chien se tient un homme capuché et masqué, dans la main duquel un autre graffeur anonyme a depuis rajouté une laisse... A-t-il complété l'œuvre de Banksy, ou bien l'a-t-il vandalisée ? Le graff étant lui-même une forme de vandalisation peut-il être vandalisé ? Dans le même ordre d'idées, une œuvre réellement ouverte, comme l'est le graff, ne l'est pas seulement du point de vue de toutes ses réceptions possibles, mais aussi du point de vue de ses transformations et détournements potentiels.

31. op. cit., p.186.

32. Les œuvres de Banksy combinent souvent images et textes en effaçant toute frontière entre les deux. Le texte chez lui ne légende pas l'image, il est absorbé par elle dans un au-delà du texte que l'on peut définir comme l'iconicité du texte, et l'image n'exhausse pas le texte, elle fait signe vers la textualité de l'image, dans un mouvement prolongé qui va des tablettes cunéiformes au writing des street artistes. 
33. Banksy fait clairement référence à Lascaux dans certaines de ses œuvres.

34. « N'y a-t-il pas, sous le même nom d'image, plusieurs fonctions dont l'ajustement problématique constitue précisément le travail de l'art? », Jacques Rancière, Le destin de l'image, Paris, Éd. La Fabrique, 2003, p.9.

35. Better Out than In, Mieux dehors que dedans, intitulé de cette performance à propos de laquelle il précise : «Il n'y a absolument aucune raison de faire cette performance..., je voulais faire de l'art sans étiquette de prix qui y soit attachée ». Cette exposition sauvage comportait des graffitis, mais aussi des installations vidéo, des sculptures et des performances.

36. A propos de l'architecture de Lucien Kroll, Jurgen Habermas, Ecrits politiques, Paris, Flammarion, 1999, p. 12.

37. cf. Le pochoir des chimpanzés affublés d'une pancarte et apostrophant les passants ainsi : « Riez maintenant, un jour nous serons aux commandes ", District Lane, Londres, 2002. Ou bien le mur demandant à la caméra de surveillance ce qu'elle est en train de regarder, Marble Arch, Londres, 2004.

38. New-York, Better Out than In.

39. On retrouve ici l'écho des injonctions husserliennes et l'apport de la phénoménologie dont la méthode visait à revenir "aux choses mêmes ». cf. Francis Ponge, Le parti pris des choses, Paris Gallimard, 1967.

40. "Ce héros anonyme vient de très loin, c'est le murmure des sociétés », Michel de Certeau, Les arts de faire, tome 1, L'invention du quotidien, Paris, Gallimard, 1990, p.11.

41. "Il y a des formes de "communauté des égaux", des manières dont une certaine égalité produit certaines formes de communautés. L'anonymat de l'art est une de ces formes. Cela ne renvoie pas à l'idée d'un créateur collectif, ou à l'idée que l'art se perdrait dans le collectif, dans la construction des formes de la vie commune, selon des modèles que l'on connaît bien de la communauté comme oeuvre d'art. C'est plutôt que les oeuvres de l'art tendent à se présenter comme des espèces de points où l'expérience s'éclaire elle-même ", Jacques Rancière, Entretien avec Yan Ciret dans Art Press, à propos de son ouvrage Le destin de l'image, Paris, Éd. La Fabrique, 2003.

42. Expression prononcée par Paul Klee et gravée sur sa tombe.

43. Postface aux Lois de l'hospitalité, Paris, Gallimard, 1995, p.335.

44. Michel Foucault, Propos introductifs à “Qu'est-ce qu'un auteur?”, op.cit.

45. Roberte, ce soir, Paris, Gallimard, 1954, p.52.

46. L'invention du quotidien, op.cit., p. XLVI.

47. Les murs parlent, ses œuvres s'interrogent à notre propos. Exemples : Banksy, Guerre et Spray, op.cit., p. 15 et p.83.

48. Privilèges des saints et des héros. A propos des héros, Banksy parle de l'invisibilité comme d'un « superpouvoir».

49. Paris, Gallimard, 1982, p.411.

50. Marie-José Mondzain, Le commerce des regards, Paris, Le Seuil, 2003.

\section{ABSTRACTS}

Refuse to be named, while pointing art as a new esthetic experience of politics, is a challenge nevertheless regularly damaged by the digital strategies of unveiling of the most famous 
unknown's name on the street-art area. With his anonymity, Banksy joins masters of the Renaissance whose artistic virtuosity was strengthened by the impossible identification of the author of the work. The analysis of its performances, the most recent to oldest, will try to understand the meaning of the agir banksyen, hollow artist and relief work.

Refuser de se nommer, tout en désignant l'art comme expérience esthétique nouvelle du politique, relève d'un défi pourtant régulièrement mis à mal par les stratégies numériques de dévoilement du nom de l'inconnu le plus célèbre de la planète street-art. Par son anonymat, Banksy rejoint les maîtres de la Renaissance dont la virtuosité artistique s'élargissait de l'impossible identification de l'auteur de l'œuvre. L'analyse de ses performances, des plus récentes ou plus anciennes, tentera de cerner le sens de l'agir banksyen, artiste en creux et œuvre en relief.

\section{INDEX}

Geographical index: France

Chronological index: XXIème siècle

Mots-clés: Banksy, street art, anonymat, esthétique, politique

\section{AUTHOR}

\section{MARIE-JOSEPH BERTINI}

Marie-Joseph Bertini est Professeur des Universités en Sciences de l'Information et de la Communication. Directrice de recherches au LIRCES (Laboratoire Interdisciplinaire Récits, Cultures et Sociétés), elle développe des travaux portant notamment sur les images et les représentations sociales et culturelles qui leur sont attachées. 